\title{
Hard arteries, weak bones
}

\author{
James F. Griffith $\cdot$ S. M. Kumta $\cdot$ Yu Huang
}

Published online: 27 February 2011

(C) ISS 2011

Given the close association between bone and blood, it is not that surprising how the two common diseases of artery and bone, namely atherosclerosis and osteoporosis, may in part share a common pathogenesis. Although atherosclerosis and osteoporosis are both clearly more prevalent in later years, there is nonetheless increasing evidence of a bone-vascular connect independently linking osteoporosis with atherosclerosis beyond the occurrence of shared risk factors such as age, gender, smoking, lifestyle factors, alcohol, and diabetes.

Several studies have shown how atherosclerosis is more common in both men and women with osteoporosis or low bone mass ('osteopenia'). Regarding cerebrovascular disease, a study of over 9,500 women aged more than 65 years, showed how low areal bone mineral density (BMDa) at baseline increased the relative risk of stroke by 1.7 with low BMDa at baseline proving to be an even stronger predictor of subsequent stroke than systolic blood pressure [1]. In over 4,300 middle-aged to elderly men and women followed-up for an average 5.6 years, relative risk of both stroke and death from any cause was almost twice as high in those with

\section{J. F. Griffith $(\bowtie)$}

Department of Imaging and Interventional Radiology,

The Chinese University of Hong Kong, Shatin,

Hong Kong

e-mail: griffith@cuhk.edu.hk

\section{S. M. Kumta}

Department of Orthopaedics and Traumatology,

The Chinese University of Hong Kong, Shatin,

Hong Kong

\section{Y. Huang}

Institute of Vascular Medicine, Li Ka Shing Institute of Health

Sciences and School of Biomedical Sciences,

The Chinese University of Hong Kong, Shatin,

Hong Kong osteoporosis at baseline even after adjusting for confounding variables such as age, smoking, diabetes, and hypertension [2]. In post-menopausal women, the decrease in lumbar BMDa is associated with an increase in carotid intimal thickness $(p<0.01)[3]$.

Regarding cardiovascular disease, low BMDa independently predicts coronary artery disease in women undergoing coronary angiography better than traditional risk factors such as age, hypertension, diabetes, smoking, family history, or dyslipidemia [4]. In a study of over 2,500 postmenopausal women followed-up for more than 4 years, not only did women with osteoporosis have a 3.9-fold increased risk for cardiovascular events compared to women with low bone mass, it was also seen that the more severe the impairment of bone strength, as evidenced by the frequency and severity of vertebral fracture, the greater the risk of a subsequent cardiovascular event [5].

For peripheral vascular disease, a study of almost 6,000 elderly male subjects, followed-up for more than 5 years, showed how BMD loss was almost twice as pronounced in those with peripheral arterial disease as those with no peripheral arterial disease while the relative risk of nonspinal fracture was also increased by 1.47 [6]. Although low-energy fractures are a more reliable indicator of osteoporosis than BMD, when addressing the link between osteoporosis and atherosclerosis, BMD or other structural bone parameters may be more apt as vascular disease per se will increase the risk of fall and thereby the likelihood of fracture.

Osteoporosis is linked to atherosclerosis and probably even more so to the occurrence of vascular calcification, which is a very frequent accompaniment of atherosclerosis. For example, in both sexes, lower lumbar vertebral trabecular BMD is more strongly associated with increasing calcification and severity of carotid atherosclerotic plaque 
than increasing intima media thickness [7]. In men, the increase in abdominal aortic calcification is associated with lower BMD and impairment of trabecular structural parameters though this did not remain significant for women after confounding variables were considered [8].

Far from being a passive process simply related to calcium-phosphate imbalance, vascular calcification is a tightly regulated process that results in extracellular matrix deposition by osteoblast cells derived from either stem cells (circulating or within the arterial wall) or through differentiation of pre-existing vascular cells such as smooth muscle cells or pericytes. The commonest types of cardiovascular calcification are atherosclerotic calcification, medial artery calcification, and cardiac valve calcification. These types of calcification evolve though fairly distinctive biological processes. Atherosclerotic calcification occurs in atherosclerotic plaques, which are composed of cellular necrosis, inflammation, and cholesterol deposits, by a process similar to endochondral ossification with deposition of a chondroid matrix prior to bone formation [9]. So advanced is the level of bone formation that even mature bone elements with marrow components may occasionally be seen in atherosclerotic plaques. Medial artery calcification, on the other hand, occurs by a process similar to intramembranous ossification without prior chondroid matrix deposition [9]. This type of vascular calcification is more florid, more visible radiographically and more common in diabetics, chronic renal failure, and the elderly. Cardiac valve calcification contains features of both atherosclerotic and medial artery calcification though is more disorganized and probably results from a combination of mechanical injury and inflammation within the cardiac valve [9]. Although no large comparative study has been performed, initial studies suggest that medial artery calcification seems the more common type of vascular calcification associated with osteoporosis.

Atherosclerosis leads to reduced bone blood flow and reduced bone perfusion. The terms 'blood flow' and 'perfusion' embody different physiological processes with 'blood flow' equating to flow within the vascular system and 'perfusion' representing a more encompassing and functionally more relevant process comprising blood flow, endothelial permeability, and interstitial diffusion. Magnetic resonance dynamic contrast-enhanced imaging provides a measure of perfusion rather than blood flow. As a rough guide, the outer one-third of the cortex (up to $5.5 \mathrm{~mm}$ thick) receives its blood supply from the periosteal arteries while the inner two-thirds of the cortex, the marrow constituents, and the trabecular bone are supplied by the nutrient arteries via the marrow cavity. Even this distribution is interesting given that osteoporosis is associated with bone deposition on the periosteal side and bone loss on the endosteal side of the cortex leading to an increase in bone cross-sectional area which helps to maintain bone strength in the presence of net bone loss. Rather than possessing a capillary network, the medullary canal comprises voluminous sinusoids lined by endothelial cells covered by a continuous basement membrane. These endothelial cells lack a tight junction though may overlap or interdigiate facilitating a two-way passage of hematopoietic cells. The thin $(50-150 \mu \mathrm{m})$ trabeculae, which are the most active component of bone, do not possess a Haversian system or capillary system though do have a fine canalicular network linking embedded osteocytes to the bone surface. Since trabeculae are dependent for their nutrition on diffusion from the adjacent sinusoids and perivascular spaces, it is conceivable how they may be particularly sensitive to changes in the composition of their immediate extraluminal microenvironment.

Age-related reduction in blood flow to bone has been observed in both humans and experimental animals [10]. Nowadays, cross-sectional imaging studies enable the accurate non-invasive quantification of bone mineral density, vascular calcification, body fat, marrow fat, and marrow perfusion. Compared to the large number of studies exploring the bone-vascular connect at a cellular level, relatively few clinical imaging studies have been performed. MR perfusion imaging studies have shown that vertebral marrow perfusion is reduced in older subjects [11]. In small animals, appendicular blood flow tends to reduce more in the diaphyses $(\downarrow 45 \%)$ with aging than the metaphyses $(\downarrow 21-28 \%)$ [10] paralleling the marrow conversion from hematopoietic to fatty marrow seen in humans. Reduced lumbar BMDa is associated with decreased bone marrow perfusion and increased marrow fat content in both males [12] and females [13] and in the proximal femur [14] as well as the lumbar spine $[12,13]$. This reduced perfusion does not appear to be reflective of a generalized circulatory disturbance as no corresponding perfusion abnormality was observed in adjacent skeletal muscle $[13,14]$. There are three likely causes of reduced bone perfusion in osteoporosis. The first is atherosclerosis leading to progressive narrowing of the intramedullary arteries as a feature of aging [15], and in line with this, MR perfusion parameters are seen to be related to arterial intima-media thickening [16]. The second is endothelial dysfunction, which is one of the earliest manifestations of atherosclerosis and aggravated by sex hormone depletion. Endothelial dysfunction leads to reduced vasodilatation and increased resistance to blood flow ('impaired vascular reactivity') and seems to occur in all arteries, including most likely the nutrient arteries of bones. Impairment of endothelium-dependent vasodilatation is closely associated with the reduced bioavailability of nitric oxide (NO), which can be caused by either the diminished activity of endothelial NO synthase (eNOS) 
and NO production or exaggerated reactive oxygen species (ROS) production (increased oxidative stress) [17]. Nitric oxide is a highly diffusible and reactive gas lasting only for a few seconds. In every systole, the arteriolar endothelium releases a small puff of $\mathrm{NO}$, which diffuses into the local vascular smooth muscle, causes vasodilatation, and thus increases blood flow. Impaired endothelial function can occur at a very young age and has, for example, been observed in young diabetics and overweight children [18]. After adjusting for age and years since the menopause, women with low bone mass or osteoporosis had significantly impaired endothelial function than those of normal BMDa [19]. Endotheliumdependent vasodilatation is about $20-25 \%$ lower in the femoral nutrient arteries of aged experimental male rats [10]. Since blood flow is directly dependent on the vessel radius to the 4th power (Poiseuille's law), a 25\% reduction of vessel diameter due to either arthrosclerosis and/or endothelial dysfunction could potentially reduce volumetric blood flow to the medullary canal by $68 \%$. The third potential cause of reduced perfusion seen in osteoporotic bone is a reduction in the amount of hematopoietic marrow accompanying osteoporosis $[15,20]$. A decrease does occur in hematopoietic marrow volume with aging and this may be accelerated in subjects with osteoporosis, though not of sufficient severity to result in clinical anemia $[15,20]$. Since the metabolic requirement of hematopoietic tissue is much greater than that of bone tissue and marrow fat, it is plausible that a reduced hematopoietic marrow volume may be driving the observed reduction in bone perfusion seen in osteoporosis [20].

As one progresses though life, two osteoporotic streams seem to be acting on bone [21]. The first is the slow gradual bone loss that begins soon after peak bone mass, and the second is the accelerated bone loss that begins later in life. Bone loss begins in the third decade in both men and women, before any appreciable reduction in sex hormone level occurs. Between the ages of 30 and 55 years, there is a gradual decrease in femoral neck BMD by about $2-4 \%$ in females and $4-6 \%$ in males per decade [22]. MR-based study has shown that over the same time period, there is a gradual increase in marrow fat also of about $5-7 \%$ per decade [23] though no associated obvious change in marrow fat composition [24]. This bone-fat transition, which is accentuated in women after the menopause, may be related to an age-related drift in mesenchymal stem cell differentiation towards adipocytic and away from osteoblastic or hematological cell lines [21].

Over and above the gradual deduction in bone mass that occurs from an early age, older males and females, though particularly females, are prone to more accelerated bone loss. There are many potential culprits for this accelerated post-menopausal bone loss with a bone-vascular connect, aggravated by sex-hormone depletion, being a plausible possibility. Bone and vasculature are so closely connected that, at a cellular level, well over a hundred potential mechanisms exist whereby arteries can interact with bone and visa versa $[25,26]$. Broadly speaking, an arterial disorder may be affecting bone; a bone disorder may be affecting arteries or both tissues may be influenced by common extraneous factor or factors.

Impaired bone perfusion due to arthrosclerosis and/or endothelial dysfunction may increase oxidative stress within the bone marrow, exacerbating a drift in mesenchymal stem cell differentiation towards adipocytosis and away from osteoblastogenesis or hematopoiesis [21]. Endothelial dysfunction itself has a potent downstream effect on bone metabolism by decreasing the local production of nitric oxide and prostaglandin $\mathrm{E}_{2}\left(\mathrm{PGE}_{2}\right)$ (both of which stimulate osteoblasts and inhibit osteoclasts), decreasing production of $\mathrm{PGI}_{2}$ (which inhibits osteoclasts) and decreasing production of the bone matrix protein osteopontin [27]. In other words, endothelial dysfunction may, through local mediators, reduce osteoblastic and increase osteoclastic activity [27]. The effect of $\mathrm{NO}$ on bone is very concentration-dependent. Low NO concentration maintains normal osteoblastic-osteoclastic function while a slightly elevated NO concentration inhibits osteoclastic activity and stimulates osteoblastic activity. Higher NO concentrations inhibit both osteoclastic and osteoblastic activity [28]. Reduced bone blood flow will lead to reduced interstitial fluid flow and reduced shear stresses among bone cells. These shear stresses stimulate the local release of bone remodeling mediators such as NO and PGI2 within the functionally important bone remodeling units and may be related to the rapid loss on bone (and muscle) mass seen in space flights. NO appears to modulate the anabolic effect that estrogen has on bone while NO metabolites are increased by estrogen replacement [28]. Statins, through an effect on mevalonic acid and subsequently Rho synthesis, help to up-regulate eNOS expression. Endothelial dysfunction is also reversed by angiotensin converting enzyme and exercise.

The process of vascular calcification involves many factors that are also important for bone mineralization (such as bone morphogenetic protein 2, Wrnts, osteopontin, matrix Gla proteins, osteocalcin, and osteoprotegerin). These proteins are present in the arterial intima and aortic valve where they are synthesized by vascular cells and regulate vascular calcification and ossification [26]. These same proteins help regulate osteoblast:osteoclast activity, bind growth factors, and assist in apatite deposition in bone [26]. While bone-modulating factors released from calcifying arteries may be influencing bone downstream, it is also conceivable that the reverse is true and mediators over spilling from bone may be secondarily affecting arteries. Bone morphogenetic protein 4 (BMP4) has emerged as an important pro-inflammatory 
molecule that is up-regulated in calcified atherosclerotic plaques [29] and impairs endothelial function through ROSdependent cyclooxygenase-2 up-regulation [30]. Although BMP4 participates in the onset of endochondral bone formation in humans, its role in bone disease in association with the change in hormonal status is still unclear. BMP4 gene polymorphism was found to affect bone mass in postmenopausal women [31] and could be another potential common regulator of vascular and bone function.

Inflammation is another process that can connect atherosclerosis and bone loss with many similarities existing in the inflammatory mechanisms that can potentially intervene between atherosclerosis and osteoporosis. This is understandable given that osteoclasts derive from the macrophage cell line in the bone marrow while in the arterial wall, macrophages are key to the development and progression of atherosclerosis [26]. For example, subendothelial accumulation of oxidized lipids, seen from the very earliest stage of plaque formation, induces osteoblast differentiation in arteries while these same oxidized lipids inhibit osteoblast formation in bone [9].

"In youth, we run into difficulty. In old age, difficulties run into us" (Beverly Sills, American soprano, 1929-2007). Atherosclerosis and osteoporosis are two very common largely clinically silent diseases of old age. One can appreciate that identification of a relevant contributory link between atherosclerosis and osteoporosis would be a significant step in the development of a single therapy to help both conditions. Nowadays, with the use of accurate non-invasive imaging techniques and advanced analytical methods, we are in a good position to help move this quest forward through identification of subclinical disease and exploring, in a wider sense, the many potential mechanisms driving the bone-vascular connect.

\section{References}

1. Browner WS, Seeley DG, Vogt TM, Cummings SR. Non-trauma mortality in elderly women with low bone mineral density. Study of Osteoporotic Fractures Research Group. Lancet. 1991;338: $355-8$.

2. Nordström A, Eriksson M, Stegmayr B, Gustafson Y, Nordström P. Low bone mineral density is an independent risk factor for stroke and death. Cerebrovasc Dis. 2010;29:130-6.

3. Sumino H, Ichikawa S, Kasama S, Takahashi T, Sakamoto H, Kumakura H, et al. Relationship between carotid atherosclerosis and lumbar spine bone mineral density in postmenopausal women. Hypertens Res. 2008;31:1191-7.

4. Marcovitz PA, Tran HH, Franklin BA, O'Neill WW, Yerkey M, Boura $\mathrm{J}$, et al. Usefulness of bone mineral density to predict significant coronary artery disease. Am J Cardiol. 2005;96: 1059-63.

5. Tankó LB, Christiansen C, Cox DA, Geiger MJ, McNabb MA, Cummings SR. Relationship between osteoporosis and cardiovas- cular disease in postmenopausal women. J Bone Miner Res. 2005;20:1912-20.

6. Collins TC, Ewing SK, Diem SJ, Taylor BC, Orwoll ES, Cummings SR, et al. Osteoporotic Fractures in Men (MrOS) Study Group. Peripheral arterial disease is associated with higher rates of hip bone loss and increased fracture risk in older men. Circulation. 2009;119:2305-12.

7. Hyder JA, Allison MA, Barrett-Connor E, Detrano R, Wong ND, Sirlin C, et al. Bone mineral density and atherosclerosis: the multiethnic study of atherosclerosis, abdominal aortic calcium study. Atherosclerosis. 2010;209:283-9.

8. Chow JT, Khosla S, Melton 3rd LJ, Atkinson EJ, Camp JJ, Kearns AE. Abdominal aortic calcification, BMD, and bone microstructure: a population-based study. J Bone Miner Res. 2008;23: 1601-12.

9. Johnson RC, Leopold JA, Loscalzo J. Vascular calcification: pathobiological mechanisms and clinical implications. Circ Res. 2006;99:1044-59.

10. Prisby RD, Ramsey MW, Behnke BJ, Dominguez 2nd JM, Donato AJ, Allen MR, et al. Aging reduces skeletal blood flow, endothelium-dependent vasodilation, and NO bioavailability in rats. J Bone Miner Res. 2007;22:1280-8.

11. Baur A, Stabler A, Bartl R, Lamerz R, Scheidler J, Reiser M. MRI gadolinium enhancement of bone marrow: age-related changes in normals and in diffuse neoplastic infiltration. Skeletal Radiol. 1997;26:414-8.

12. Griffith JF, Yeung DK, Antonio GE, Lee FK, Hong AW, Wong SY, et al. Vertebral bone mineral density, marrow perfusion, and fat content in healthy men and men with osteoporosis: dynamic contrast-enhanced MR imaging and MR spectroscopy. Radiology. 2005;236:945-51.

13. Griffith JF, Yeung DK, Antonio GE, Wong SY, Kwok TC, Woo J, et al. Vertebral marrow fat content and diffusion and perfusion indexes in women with varying bone density: MR evaluation. Radiology. 2006;241:831-8.

14. Griffith JF, Yeung DK, Tsang PH, Choi KC, Kwok TC, Ahuja AT, et al. Compromised bone marrow perfusion in osteoporosis. J Bone Miner Res. 2008;23:1068-75.

15. Demmler K, Otte P, Bartl R, et al. Osteopenia, marrow atrophy and capillary circulation: comparative studies of the human iliac crest and 1st lumbar vertebra. Orthop. 1983;121:223-7.

16. Chen WT, Shih TT, Hu CJ, Chen RC, Tu HY. Relationship between vertebral bone marrow blood perfusion and common carotid intima-media thickness in aging adults. J Magn Reson Imaging. 2004;20:811-6.

17. Wong WT, Wong SL, Tian XY, Huang Y. Endothelial dysfunction: the common denominator in hypertension and diabetes. J Cardiovasc Pharmacol. 2010;55:300-7.

18. Khan F, Green FC, Forsyth JS, Greene SA, Morris AD, Belch JJ. Impaired microvascular function in normal children: effects of adiposity and poor glucose handling. J Physiol. 2003;551:705-11.

19. Sumino H, Ichikawa S, Kasama S, Takahashi T, Sakamoto H, Kumakura $\mathrm{H}$, et al. Relationship between brachial arterial endothelial function and lumbar spine bone mineral density in postmenopausal women. Circ J. 2007;71:1555-9.

20. Griffith JF, Wang YX, Zhou H, Kwong WH, Wong WT, Sun YL, et al. Reduced bone perfusion in osteoporosis: likely causes in an ovariectomy rat model. Radiology. 2010;254:739-46.

21. Rosen CJ, Ackert-Bicknell C, Rodriguez JP, Pino AM. Marrow fat and the bone microenvironment: developmental, functional, and pathological implications. Crit Rev Eukaryot Gene Expr. 2009;19:109-24.

22. Lynn HS, Lau EM, Au B, Leung PC. Bone mineral density reference norms for Hong Kong Chinese. Osteoporos Int. 2005; $16: 1663-8$. 
23. Kugel H, Jung C, Schulte O, Heindel W. Age- and sex-specific differences in the $1 \mathrm{H}$-spectrum of vertebral bone marrow. J Magn Reson Imaging. 2001;13:263-8.

24. Griffith JF, Yeung DK, Ahuja AT, Choy CW, Mei WY, Lam SS, et al. A study of bone marrow and subcutaneous fatty acid composition in subjects of varying bone mineral density. Bone. 2009;44:1092-6.

25. Demer LL, Tintut Y. Mechanisms linking osteoporosis with cardiovascular calcification. Curr Osteoporos Rep. 2009;7:42-6.

26. Hamerman D. Osteoporosis and atherosclerosis: biological linkages and the emergence of dual-purpose therapies. QJM. 2005;98:467-84.

27. Bloomfield SA, Hogan HA, Delp MD. Decreases in bone blood flow and bone material properties in aging Fischer-344 rats. Clin Orthop. 2002;396:248-57.
28. Wimalawansa SJ. Nitric oxide and bone. Ann NY Acad Sci. 2010;1192:391-403.

29. Vendrov AE, Madamanchi NR, Hakim ZS, Rojas M, Runge MS. Thrombin and $\mathrm{NAD}(\mathrm{P}) \mathrm{H}$ oxidase-mediated regulation of $\mathrm{CD} 44$ and BMP4-Id pathway in VSMC, restenosis, and atherosclerosis. Circ Res. 2006;98:1254-63.

30. Wong WT, Tian XY, Chen YC, Leung FP, Liu LM, Lee HK, et al. Bone morphogenic protein-4 impairs endothelial function through oxidative stress-dependent cyclooxygenase-2 upregulation: implications on hypertension. Circ Res. 2010;107:984-91.

31. Ramesh Babu L, Wilson SG, Dick IM, Islam FM, Devine A, Prince RL. Bone mass effects of a BMP4 gene polymorphism in postmenopausal women. Bone. 2005;36:555-61. 